\title{
Awareness Science and Engineering
}

\author{
Qiangfu Zhao, ${ }^{1}$ Cheng-Hsiung Hsieh, ${ }^{2}$ Keitaro Naruse, ${ }^{1}$ and Zhishun She ${ }^{3}$ \\ ${ }^{1}$ School of Computer Science and Engineering, The University of Aizu, Aizuwakamatsu 965-8580, Japan \\ ${ }^{2}$ Department of Computer Science and Information Engineering, Chaoyang University of Technology, Taichung 41349, Taiwan \\ ${ }^{3}$ Department of Electrical Engineering and Computer Science, Glyndwr University, Wrexham LL11 2AW, UK \\ Correspondence should be addressed to Qiangfu Zhao, qf-zhao@u-aizu.ac.jp
}

Received 7 June 2012; Accepted 7 June 2012

Copyright (C) 2012 Qiangfu Zhao et al. This is an open access article distributed under the Creative Commons Attribution License, which permits unrestricted use, distribution, and reproduction in any medium, provided the original work is properly cited.

The goal of awareness computing (AC) is to realize awareness in computing machines. Awareness is the ability to perceive, to feel, or to be conscious of events, objects, or sensory patterns. It may not lead directly to full comprehension. Awareness often implies vigilance in observing and alertness in drawing inferences from what one experiences. Compared with artificial intelligence (AI), the goal of $\mathrm{AC}$ is less ambitious. Nevertheless, AC could be more important for solving practical problems. We think that awareness is the bridge between perception and cognition. Without being aware, a system may never become intelligent. Moreover, awareness could make computation towards the goal of AI more efficient by shedding of irrelevant possibilities.

In the last two decades, $\mathrm{AC}$ has been studied mainly from an engineering perspective. To create real aware systems, however, studying different applications in an ad hoc manner is not enough. We need to study all kinds of AC-related problems in a unified framework and gain more insight about aware systems and the mechanism of awareness existing in different living beings. For this purpose, we have organized the first International Workshop on Aware Computing (IWAC2009, Aizuwakamatsu, Japan), the second International Symposium on Awareness Computing (ISAC2010, Tainan, Taiwan), and the third International Conference on Awareness Science and Technology (iCAST2011, Dalian, China). We have also established the Technical Committee on Awareness Computing under the umbrella of the IEEE Systems, Man, and Cybernetics Society.

In this special issue, we received 23 submissions on different topics of AC. From them, 11 papers are highly recommended by the referees. We can classify these papers into 3 categories. The first category is related to the fundamental theory and modeling of AC (papers by H. Takagi entitled "Interactive evolutionary computation for analyzing human awareness mechanisms," M. Hu and H. Liang entitled "Variance entropy: a method for characterizing perceptual awareness of visual stimulus," Y. Sawada entitled "The aspects, the origin, and the merit of aware computingsuggestions from the visual hand tracking experiments," and M. Yokota entitled "Aware computing in spatial language understanding guided by cognitively inspired knowledge representation"). The second one is about realization and implementation of different AC systems (papers by M. Matsuhara and S. Suzuki entitled "Effectiveness of contextaware character input method for mobile phone based on artificial neural network," $H$. Kato et al. entitled "A realtime angle and illumination aware face recognition system based on artificial neural network," X. Yu et al. entitled "An application of improved Gap-BIDE algorithm for discovering access patterns," K. Madani "Multi-level cognitive machinelearning based concept for Artificial Awareness: application to humanoid robot's awareness using visual saliency," and J.F. Wang et al. entitled "Emotion-aware assistive system for humanistic care based on the orange computing concept"). The third is related to AC applications (papers by $\mathrm{S}$. Kikuchi and G. Chakraborty entitled "An efficient genome fragment assembling using GA with neighborhood aware fitness function" and X. Guo et al. entitled "Environmental sound recognition using time-frequency intersection patterns"). In this issue we put more weight on the scientific aspect of AC, rather than simple applications. In fact, even the two application papers are not just sensor integrations. By doing so, we hope this issue can serve as an important reference in this emerging field and provide a better understanding in establishment of a unified framework for AC. 
In the paper written by $\mathrm{H}$. Takagi entitled "Interactive evolutionary computation for analyzing human awareness mechanisms," the author attempts to analyze human awareness mechanism and to build awareness models using interactive evolutionary computation (IEC). From the related history of computational intelligence, the author paves a way to the objective. By several successful examples of IEC, the author is convinced that IEC is a possible way to awareness science. In fact, IEC can be a general tool for modeling different human-factor-related awareness.

In the paper by M. Hu and H. Liang entitled "Variance entropy: a method for characterizing perceptual awareness of visual stimulus," the authors propose a simple but efficient complexity measure called variance entropy, which can be important for characterizing perceptual awareness of visual stimulus. In the variance entropy both sample entropy and variance of data are considered. To show its effectiveness, the variance entropy is applied to analyze cortical local field potential data and to study neural dynamics of perceptual awareness. Results show that the variance entropy analysis is able to differentiate the perceptual visibility and is of far better discriminative performance than the sample entropy.

In the paper entitled "The aspects, the origin, and the merit of aware computing-suggestions from the visual hand tracking experiments," Y. Sawada studies awareness in a science perspective. He investigates several interesting aspects of awareness, including qualitative and quantitative, external and internal; awareness of thinking; awareness and experience; awareness and self-monitoring. The author also poses some profound questions for future study. For example, if a computer or robot is so aware that it can conduct self-control and self-improve based on its experiences; can we say that the computer or robot has free will? Or put it in another way, for a computer or robot to have free will, what kind of awareness should it have? After all, what is free will?

The paper written by M. Yokota entitled "Aware computing in spatial language understanding guided by cognitively inspired knowledge representation," is related to spatial relation awareness of objects in a given image, which is useful for multimedia retrieval. This paper, however, is not just an application paper. It introduces an omni-sensory mental image model and its description language Lmd. The language Lmd can provide multimedia expressions with intermediate semantic descriptions in predicate logic. This paper presents systematic and efficient computing guided by Lmd expression and 3D map data in crossmedia operation between linguistic and pictorial expressions as spatial language understanding.

In the paper by M. Matsuhara and S. Suzuki entitled "Effectiveness of context-aware character input method for mobile phone based on artificial neural network," the authors provide a character input approach for mobile phones by a context-aware mechanism. With artificial neural networks (ANNs), the proposed system becomes aware of the mapping between number segments through learning. This leads to a possibility for the system to translate the number string into the intended sentence by ANN without a dictionary. The effectiveness and feasibility of the proposed approach are verified by Twitter data on a mobile phone platform.
The paper by $\mathrm{H}$. Kato et al. entitled " A real-time angle and illumination aware face recognition system based on artificial neural network," deals with the variations of angle and illumination in the problem of face recognition. One or two multilayer perceptrons (MLPs) are trained to map angle and illumination features to image features. By the generalization ability of MLP, the variations of angle and illumination have been taken care of. Consequently, the user awareness by face images is achieved where variations of angle and illumination are considered. The approach is justified by examples.

In the paper by X. Yu et al. entitled "An application of improved Gap-BIDE algorithm for discovering access patterns," the authors propose a new algorithm for discovering access patterns. The problem studied here is related to user awareness, abnormal awareness, and is an important issue in all kinds of Internet-based service systems. Compared with the previous algorithm, a process of getting a large event set is proposed in the improved Gap-BIDE algorithm. The proposed approach can find out the frequent events by discarding the infrequent events which do not occur continuously in an accessing time before generating candidate patterns.

In the paper entitled "Multi-level cognitive machinelearning based concept for artificial awareness: application to humanoid robot's awareness using visual saliency," K. Madani et al. study the possibility of realizing artificial awareness in a robot based on observations in human early-age skill development and early-age awareness maturation. For this purpose, a multilevel cognitive procedure is introduced. Following this procedure and the proposed algorithm, a robot can percept motion, be aware of the environment visually, and pay attention to an interesting object.

In the paper by J.-F. Wang et al. "Emotion-aware assistive system for humanistic care based on the orange computing concept," studies emotion awareness and its application to mental care. In fact, mental care is one of the main objectives of the so-called orange computing. Its main purpose is to help people, not only physically but also mentally, to reduce different mental diseases which are often difficult to heal in the modern societies. In this paper, a case study on a humanmachine interactive and assistive system for emotion care is conducted. The system can detect emotional states of users by analyzing their facial expressions, emotional speeches, and laughter in a ubiquitous environment. In addition, the system can provide corresponding feedback to users according to the results.

In the paper by S. Kikuchi and G. Chakraborty entitled "An efficient genome fragment assembling using GA with neighborhood aware fitness function," the authors propose an interesting algorithm for solving the genome fragment assembling problem. The neighbor aware fitness function, although simple, is very efficient and effective for solving this NP-hard problem. Thus, different kinds of awareness are important not only for producing more intelligent systems, but also for solving different problems more intelligently. The point is that how to be aware of the necessary awareness types for solving a given problem.

In the paper by X. Guo et al. entitled "Environmental sound recognition using time-frequency intersection patterns," 
the authors provide a method that can be useful for a patrol robot to be situationally aware even in a dark environment. In this paper, a two-stage method for environmental sound recognition using neural networks (NNs) is proposed. It includes a classification stage and a recognition stage. At the classification stage, the environmental sounds are classified into three categories based on their long-term powervariance patterns, and the recognition stage recognizes the sound type based on both the short-term power-variance pattern and the instantaneous spectrum at the power peak.

Qiangfu Zhao Cheng-Hsiung Hsieh Keitaro Naruse Zhishun She 

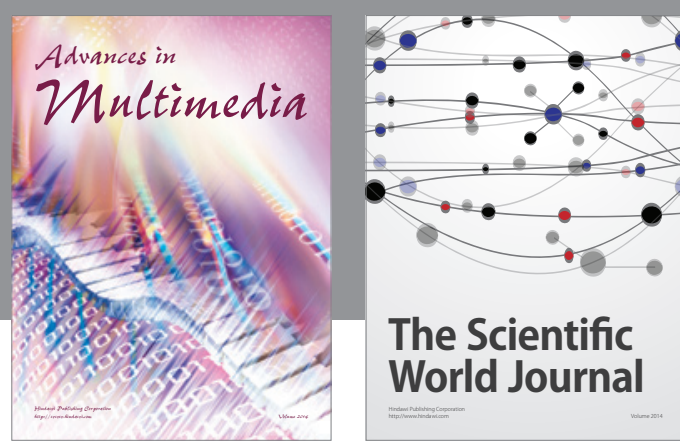

The Scientific World Journal
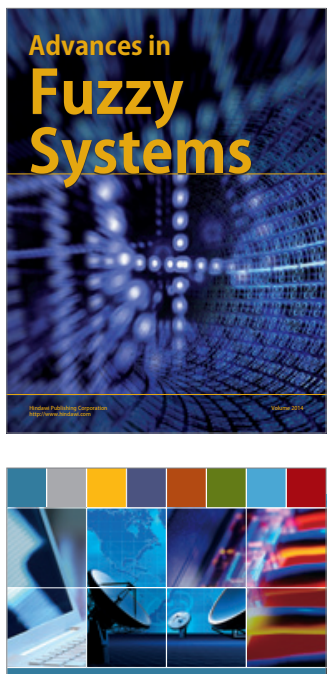

Computer Networks and Communications
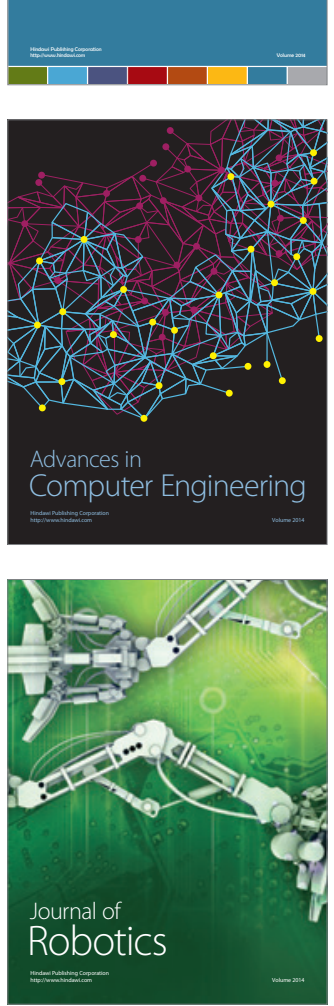
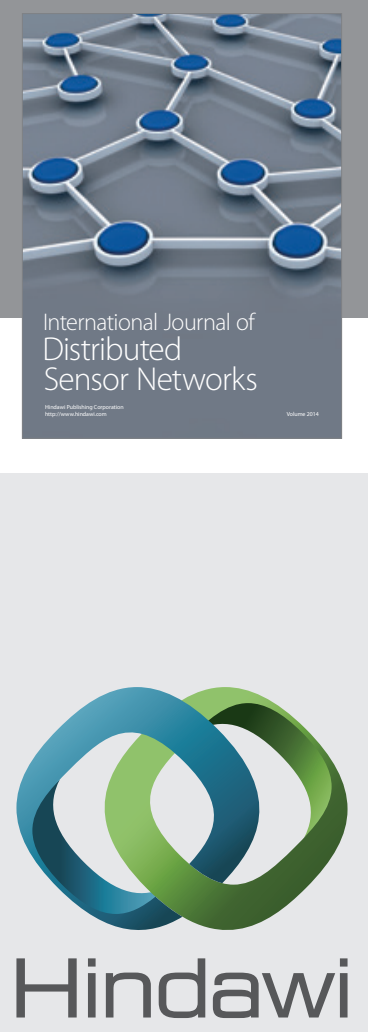

Submit your manuscripts at

http://www.hindawi.com
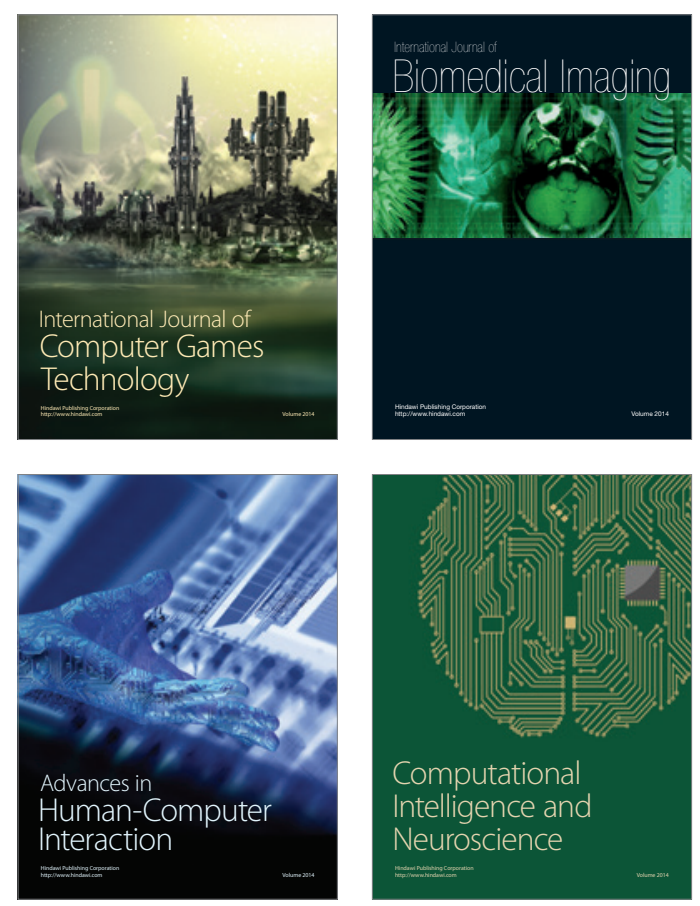
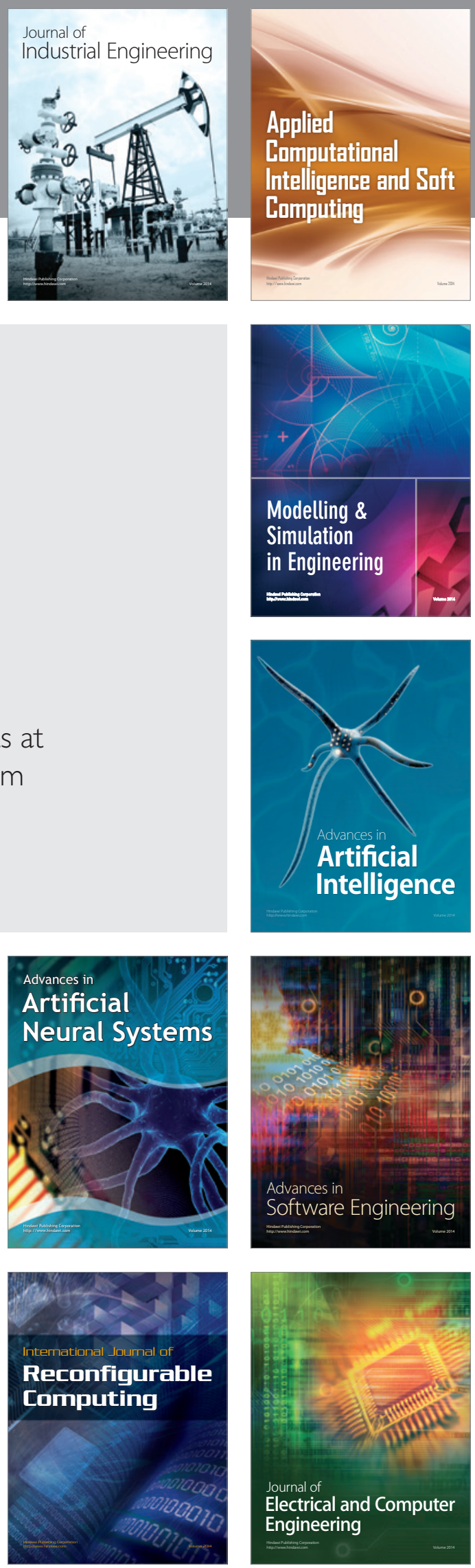